\title{
Ten Years' Survival in Patients with Cervical Cancer and Related Factors in West Azerbaijan Province: Using of Cox Proportion Hazard Model
}

\author{
Hamid Reza Khalkhali ${ }^{1}$, Rasool Gharaaghaji ${ }^{1}$, Rohollah Valizadeh ${ }^{2}$, Zahra \\ Kousehlou ${ }^{3}$, Haleh Ayatollahi ${ }^{4 *}$
}

\begin{abstract}
Objective: There are two basic ways to analyze survival data including nonparametric and parametric methods. Considering that cervical cancer is the fourth most common cancer among women, this study was conducted using a suitable regression model for survival data for patients with cervical cancer in Urmia. Material and Methods: In this historical retrospective study, all patients with cervical cancer who referred to Motahhari Hospital of Urmia during 2004 and 2015 were included in the study and consisted of 109 women with cervical cancer. The data were collected using checklist which filled with records of patients. The data were described with percent, mean and standard deviation. The survival function was computed with the Kaplan-Meier method. The adjusted Hazard Ratio for variables were estimated after fitting the Cox proportion hazard model using Forward Stepwise Likelihood Ratio method with $\mathrm{PE}=0.1$, $\mathrm{PR}=0.15$. Results: Of the 109 patients, the mean (SD) time of diagnosis was $50.1 \%(11.7)$ years. The mean (SD) and median of follow-up time was 38.23 (32.50) and 27.1 months (Mim:2 months and Max:132 months). Ninty-Three of patients $(88.7 \%)$ were in urban area. Using Log-Rank test, the mean score of survival in patients with recurrence of 38.8 months was significantly lower than those without recurrence $(102.5$ months $)(\mathrm{P}<0.001)$. The results of the COX model showed that the adjusted relative risk of mortality with a diagnostic age of 50 years or more compared to those under 50 years of age was 1.978 . The risk for marriage under age 20 was 3.189 compared to the marriage age of 20 years or more. Conclusion: According to results, the low age of marriage increases the occurrence of advanced stages of cancer in older ages and as a social and cultural factor has a significant effect on the survival of patients with cervical cancer.
\end{abstract}

Keywords: Survival analysis- cervical cancer- Cox Proportion Hazard model- West Azerbaijan Province

Asian Pac J Cancer Prev, 20 (5), 1345-1351

\section{Introduction}

According to the World Health Organization (WHO), cervix was the second most common cancer in the reproductive system of the female genitalia in 2004 and about half of million new cases of this cancer are diagnosed annually in the world, so that more than half of them lose their life (World Health Organization., 2002). Eighty percent of the people with this cancer are in developing countries (Schmoll et al., 2009). Two out three of these cases are identified in advanced stages of the disease, with a very low chance of survival (Bazaz et al., 2017). In developing countries, the rate of cervical cancer and its mortality are much higher than advanced countries (Ferlay J., 2004). Unfortunately, there are no accurate statistics about the incidence of cervical cancer in Iran, but in different reports, the annual amount was about 6-7 cases per 100,000 persons (De Martel et al., 2012, Siegel et al., 2015). According to global reports, mortality rates are higher in the Asian and Pacific countries, as in 2009, from 551 patients with cervical cancer, 211 patients died in the same year in Iran (De Martel et al., 2012).

The mean age of patients with cervical cancer is 52.2 and its age distribution is bicameral, so that its peak is between 35-39 years and 60-64 years (Vaisy et al., 2012). There were approximately 528,000 new cases of cancer occurred in the world in 2012. In less developed countries, $12 \%$ of all cancers in women consisted of cervical cancer and nearly 9 out of 10 deaths from this cancer in the world are related to these countries. The least cervical cancers are in Australia / New Zealand and the Western Asia, but it is remained the most common cancer among women in the east and middle of Africa. The estimated deaths from this cancer in worldwide were 266,000 cases in 2012 (World Health Organization, 2013).

Various causes have been reported for cervical cancer 
including long-term oral contraceptive use, low age at first intercourse, low age at first delivery, having a lot of sexual partners throughout life, having a lot of term pregnancy, high parity, smoking, illiteracy, low literacy, human papillomavirus, low socioeconomic status, etc. (Azadi et al., 2011). The vulnerable group of society against cervical cancer include: elderly people, people with low income and no insurance, but it is still controversial how they interact with these factors of cervical cancer and which of these factors have a huge impact on screening, diagnosis and survival of patient (Federico et al., 2010).

Determining the survival rates of patients with cancer is one of the ways that contribute community health workers to the better development of health care services, the implementation of cancer-related programs and the assessment of the impact of new therapies. Studies not only reveals the burden of disease in society, but also assesses the relationship between different factors with survival rates in patients (Semnani et al., 2008). In fact, survival Rate is the calculation of the survivability rate of patients from the first date of diagnosis to death time (Yazdanbod et al., 2005). In different Studies about survival rates in cervical cancer, some factors have been reported as important factors associated with survival such as the high age of diagnosis, repeated infections with the human papillomavirus virus and histology of tumor (Federico et al., 2010; Hosseini et al., 2012; Roshany et al., 2012; Serur et al., 1995).

So far, no study has been done with the aim of explaining the appropriate model for survival analysis in patients with cervical cancer in West Azerbaijan. Therefore, in this research, after fitting any of the regression models to the observations and comparing them, a suitable and effective regression model for data about survival time of patients with cervical cancer referred to Motahhari Hospital of Urmia city was performed and the role of determinants was identified.

\section{Materials and Methods}

In this historical retrospective study, all patients with cervical cancer who referred to Motahhari Hospital of
Urmia during 2004 and 2015 were included in the study and consisted of 109 women with cervical cancer and have been followed up. The data were collected using checklist which filled with records of patients. It should be mentioned that the only referral center for cervical cancer in West Azerbaijan is Shahid Motahhari Educational Center in Urmia city.

People who were not native in West Azerbaijan province, were excluded from this study. The survival time variable in this study was from diagnosis to death. Therefore, people who were alive until the end of the study or were death for non-cancer reason or excluded for any reason, considered as censored observations. Survival of patients were analyzed using the best fitted statistical model with the studied factors (age, history of surgery, history of pap smear, time of last treatment, stage of diagnosis, number of wife, recurrence of disease, tumor grade, smoking, tumor type, tumor size, cervical cancer, number of marriages, history of radiotherapy, time of first treatment).

\section{Statistical Analysis}

The survival function of patients was estimated using Kaplan-Meier method. To determine the risk factors, Log-Rank test was used to investigate the effect of each of the variables and the adjusted effects of the important factors in univariate analysis of patients' survival with Cox proportional regression model using Forward Stepwise Likelihood Ratio Method with $\mathrm{PE}=0.1, \mathrm{PR}=0.15$ were determined. Data were analyzed using SPSS / Win 22 software. There was no intervention in this study and the required data were extracted from the patients' records using a checklist without mentioning the name. In all stages of the research, the secrets of patients remained confidential and the results of the study were reported in group.

\section{Results}

Of the 109 patients, the mean (SD) time of diagnosis was $50.1 \%(11.7)$ years. The mean (SD) and median of follow-up time was 38.23 (32.50) and 27.1 months (Mim:2

\section{Survival Function}

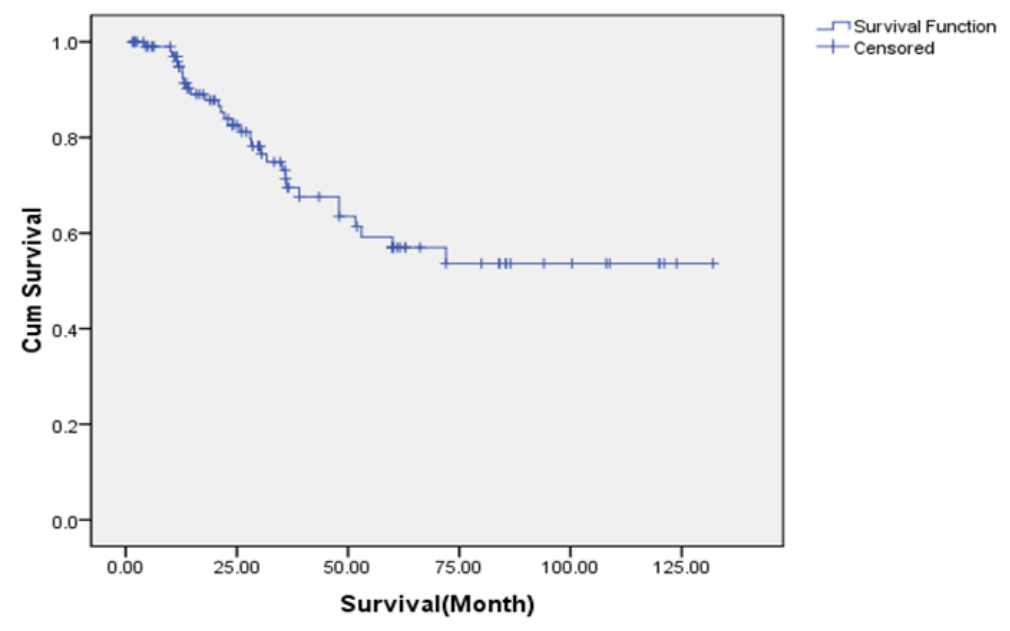

Figure 1.Survival Function of Patinas with Cervical Cancer 
Table 1. Demographic Data of Patients with Cervical Cancer

\begin{tabular}{|c|c|c|}
\hline Variable & Frequency & Percent \\
\hline \multicolumn{3}{|l|}{ Place of residence } \\
\hline Urban & 93 & 85.3 \\
\hline Rural & 16 & 14.7 \\
\hline \multicolumn{3}{|l|}{ Age at diagnosis } \\
\hline$<50$ & 49 & 45 \\
\hline$>=50$ & 60 & 55 \\
\hline \multicolumn{3}{|l|}{ Age of marriage } \\
\hline$>=20$ & 39 & 35.8 \\
\hline$<20$ & 70 & 64.2 \\
\hline \multicolumn{3}{|l|}{ Time of marriage } \\
\hline 1 & 95 & 87.2 \\
\hline$\leq 2$ & 14 & 12.8 \\
\hline \multicolumn{3}{|l|}{ Histology Type } \\
\hline $\mathrm{SCC}^{*}$ & 99 & 90.8 \\
\hline Adenocarcinoma & 10 & 9.2 \\
\hline \multicolumn{3}{|l|}{ Recurrence of the disease } \\
\hline No & 76 & 69.7 \\
\hline Yes & 33 & 30.3 \\
\hline \multicolumn{3}{|l|}{ Radiotherapy } \\
\hline No & 92 & 84.4 \\
\hline Yes & 17 & 15.6 \\
\hline \multicolumn{3}{|l|}{ FIGO Stage } \\
\hline I & 43 & 39.4 \\
\hline II & 47 & 43.1 \\
\hline III+VI & 19 & 17.4 \\
\hline \multicolumn{3}{|c|}{ Follow up For all patients (month) } \\
\hline Total N( $\mathrm{N}$ of Events) & $109(30)$ & \\
\hline Mean (SD) & $37.3 \pm 32.0$ & \\
\hline Median & 27.1 & \\
\hline Min-Max & $2-132$ & \\
\hline
\end{tabular}

*, Squamous cell carcinoma months and Max:132 months). Ninty-Three of patients (88.7\%) were in urban area. The mean (standard deviation) and median age of the marriage were 18.9 (4.00) and 18 years of old which 70 patients $(64.2 \%)$ were married at the age of less than 20 years.

Ninety-five women patients $(87.2 \%)$ were married once and 14 patients $(12.8 \%)$ at least twice. Also, among their husbands, 87 persons $(79.8 \%)$ were married once and 22 persons $(20.2 \%)$ at least twice. The mean (SD), median, minimum and maximum age of menarche were 14.27 (1.5), 14, 11 and 18 years, respectively. Only 4 patients $(7.3 \%)$ were smoker, 71 patients $(65.1 \%)$ were non-smoker and 34 (31.2\%) were unknown because there was no any information in their records.

In terms of the type of cancer histology, 99 patients (90.8\%) were Squamous cell carcinomas (SCC) and 10 patients $(9.2 \%)$ were adenocarcinoma. In terms of the recurrence, 33 patients $(30.3 \%)$ were reported with recurrence, and 76 patients $(69.7 \%)$ without recurrence. Totally, 92 patients $(84.4 \%)$ were undergone radiotherapy and 17 patients $(15.6 \%)$ did not receive radiotherapy.

According to FIGO tumor classification, 43 patients $(39.4 \%)$ had grade I, 47 patients $(43.1 \%)$ had Grade II and 19 patients (17.4\%) had Grade III and higher. During the study, 30 patients $(27.5 \%)$ died and 79 patients $(73.5 \%)$ were considered as censoring observations from the right. Summary of results is presented in Table 1.

Estimates of survival rate of six months and one, three, five and seven years were 99\%, 96.9\%, 85.2\%, 73.1\% and $57.7 \%$, respectively. The mean (SD) survival time of patients with $95 \%$ confidence intervals were 86.31 (6.46) and $(98.9,73.6)$ months, respectively (Figure 1).

In univariable analysis, the risk factors for survival time in cervical cancer patients were evaluated using log-rank test. Meanwhile, the mean survival time of patients with recurrence was 38.8 months and significantly reduced compared to non-recurrence patients with 102.5 months $(\mathrm{P}<0.001)$. The mean survival time of patients aged less than 20 years was 73.8 months, which was significantly different with patients aged twenty and over with 117.3 months $(\mathrm{P}=0.017)$.

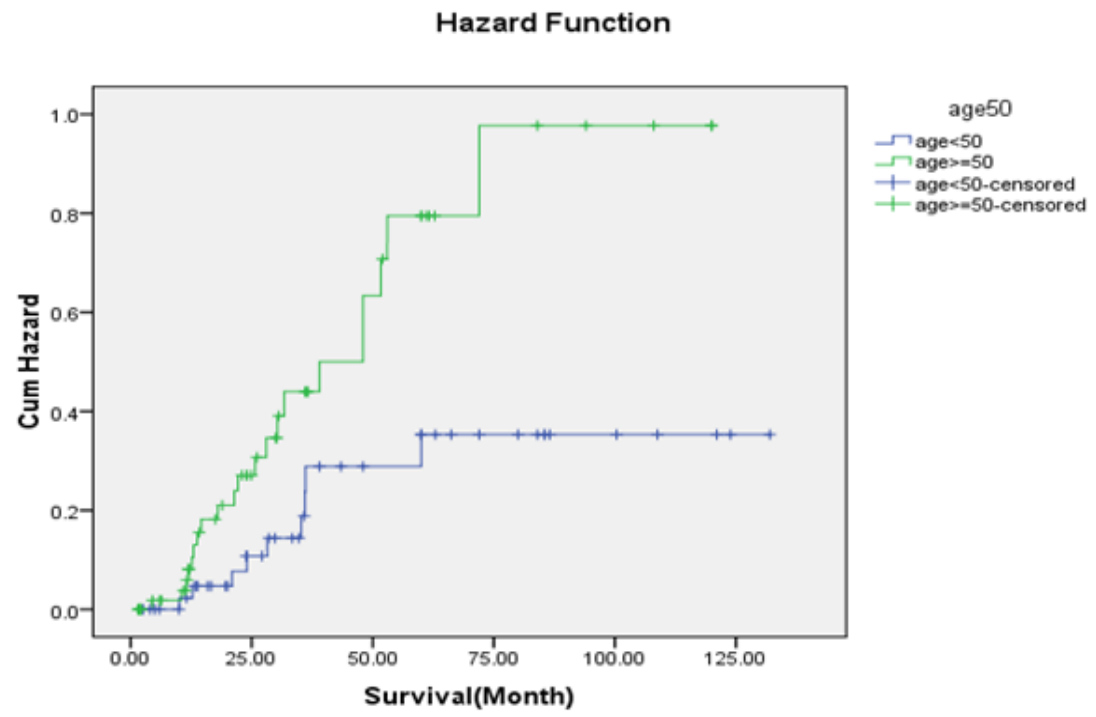

Figure 2. Hazard Function by Age 
Table 2. Means of Survival Time by Risk Factor Variables (Log-Rank Test)

\begin{tabular}{|c|c|c|c|c|c|}
\hline Variable & & Total N & $\mathrm{N}$ of Events & Means (S.D) of Survival Time & Log Rank Test Sig. \\
\hline \multirow[t]{2}{*}{ Place of residence } & Urban & 93 & 26 & $77.5(6.9)$ & \\
\hline & Rural & 16 & 4 & $59.6(6.6)$ & 0.68 \\
\hline \multirow[t]{2}{*}{ Time of marriage } & 1 & 95 & 25 & $86.9(7.1)$ & 0.8 \\
\hline & $2+$ & 14 & 5 & $73.3(12.3)$ & \\
\hline \multirow[t]{2}{*}{ Type of cancer } & $\mathrm{SCC}^{*}$ & 99 & 28 & $85.5(6.9)$ & \\
\hline & Adenocarcinoma & 10 & 2 & $75.3(13.7)$ & 0.54 \\
\hline \multirow[t]{2}{*}{ Recurrence of the disease } & No & 76 & 10 & $102.5(5.9)$ & $<0.001$ \\
\hline & Yes & 33 & 20 & $38.8(7.8)$ & \\
\hline \multirow[t]{2}{*}{ Radiotherapy } & Yes & 92 & 23 & $89.8(7.1)$ & 0.35 \\
\hline & No & 17 & 7 & $53.7(7.7)$ & \\
\hline \multirow[t]{2}{*}{ Age } & $<50$ & 49 & 9 & $102.4(8.5)$ & 0.019 \\
\hline & $>=50$ & 60 & 21 & $66.7(7.9)$ & \\
\hline \multirow[t]{2}{*}{ Age of marriage } & $>=20$ & 39 & 3 & $117.3(8.1)$ & \\
\hline & $<20$ & 70 & 27 & $73.8(6.8)$ & 0.017 \\
\hline \multirow[t]{3}{*}{ Stage } & I & 43 & 10 & $88.1(9.1)$ & \\
\hline & II & 47 & 11 & $94.7(9.3)$ & 0.013 \\
\hline & III and VI & 19 & 9 & $41.2(6.5)$ & \\
\hline
\end{tabular}

*, Squamous cell carcinoma

The diagnosis age less than 50 years with mean survival time of 102.4 months was significantly different with 50 years and over $(66.7$ months $)(P=0.019)$. Also, the mean survival time of patients with gradeI: 88.1 months, grade II: 94.7 months and grade III, and over: 41.2 months, had statistically significant difference $(\mathrm{P}=0.013)$.

\section{Hazard Function}

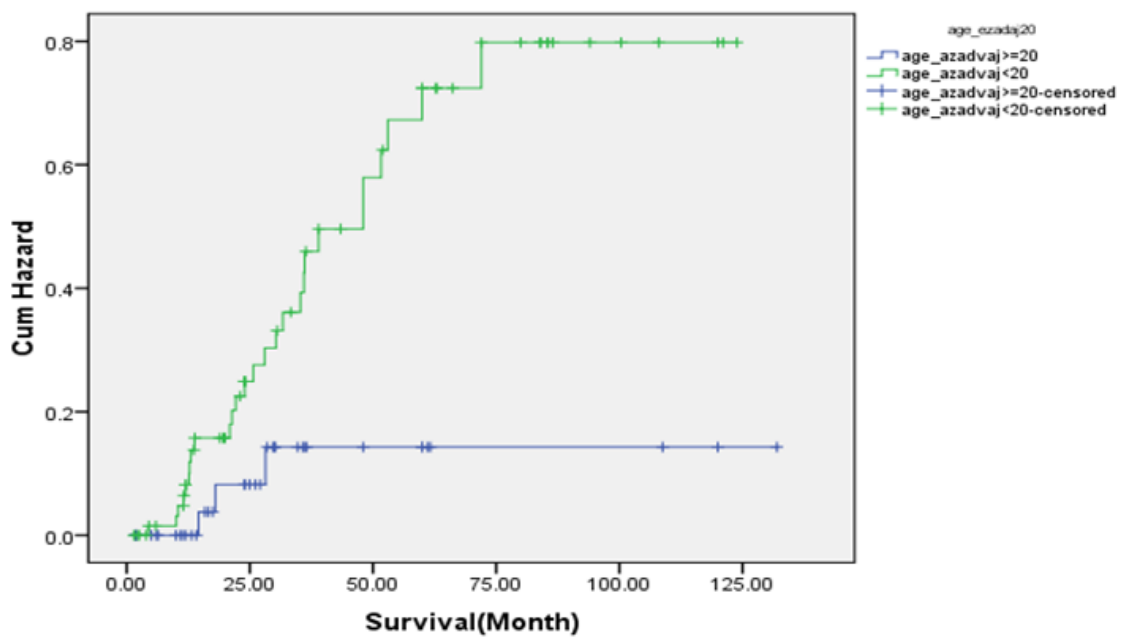

Figure 3. Hazard Function by Age in Marriage

Table 3. Factors Affecting Survival of Cervical Cancer (Multivariate Analysis)

\begin{tabular}{llccccc}
\hline variable & & B & SE & Sig. & Adjusted HR & $90.0 \%$ CI \\
\hline Age & $<50$ & Reference & - & - & - & - \\
Age of marriage & $>=50$ & 0.632 & 0.406 & 0.093 & 1.978 & $(1.014,3.858)$ \\
& $>=20$ & Reference & - & - & - & - \\
Stage & $<20$ & 1.16 & 0.615 & 0.059 & 3.189 & $(1.161,8.763)$ \\
& I & Reference & - & - & - & - \\
Recurrence of the disease & II & 0.004 & 0.438 & 0.992 & 1.004 & $(0.489,2.064)$ \\
& III and VI & 1.035 & 0.461 & 0.036 & 2.814 & $(1.318,6.010)$ \\
& No & Reference & - & - & - & - \\
& Yes & 1.89 & 0.401 & $<0.001$ & 6.618 & $(3.423,12.795)$ \\
\hline
\end{tabular}

1348 Asian Pacific Journal of Cancer Prevention, Vol 20 
Hazard Function
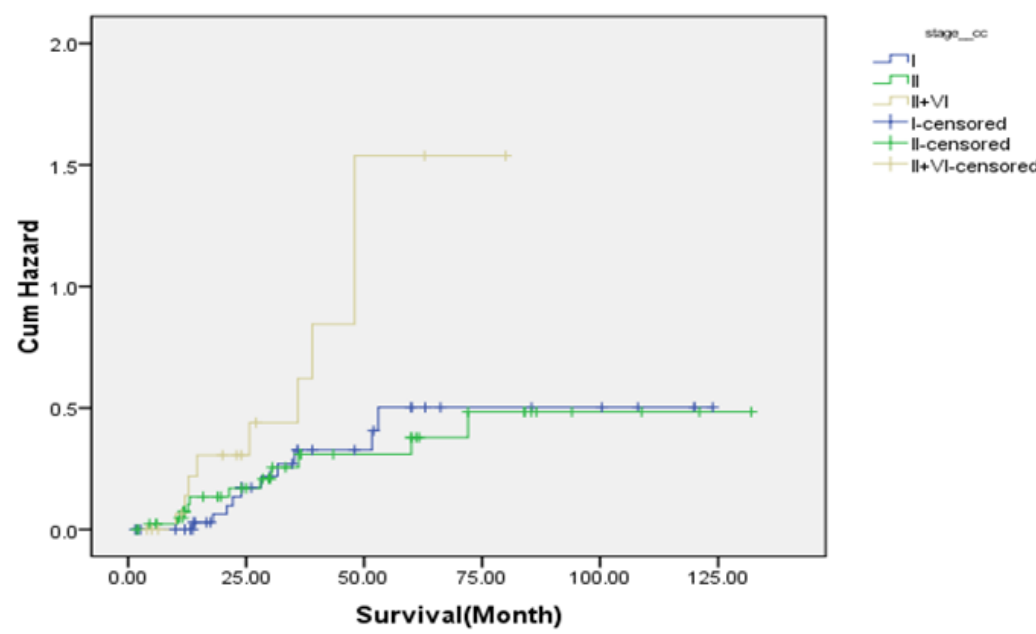

Figure 4. Hazard Function by Stage Type

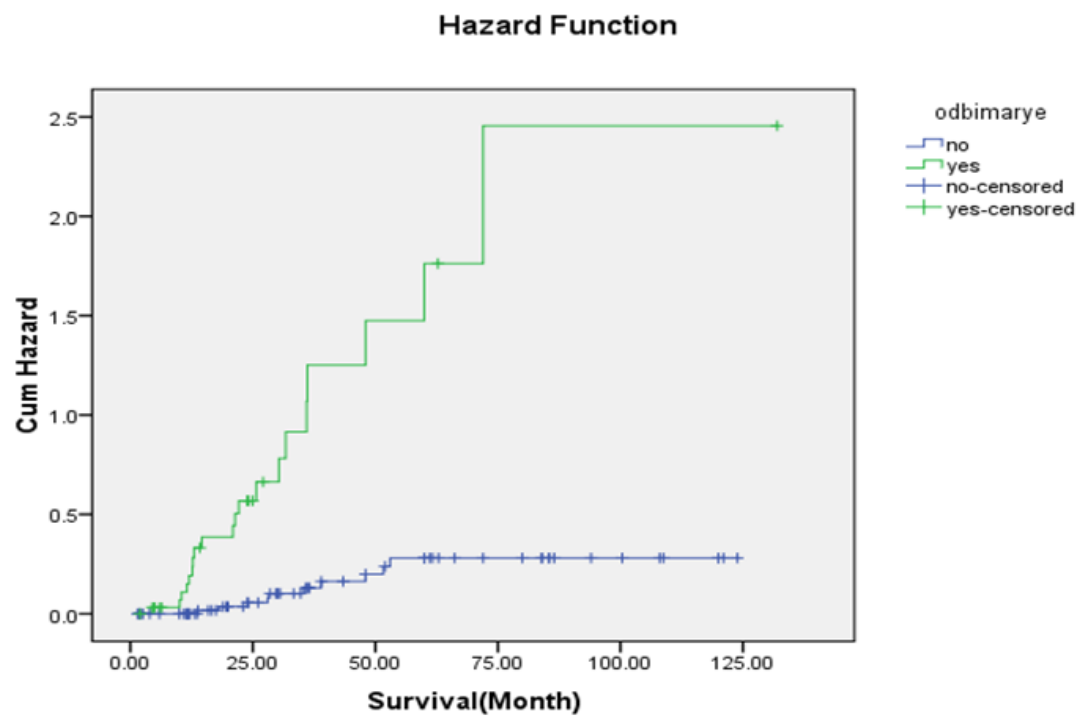

Figure 5. Hazard Functio by Odbimarye

The place of the reeisdence, having 2 times or more marriage, the type of cancer and radiotherapy treatment did not affect the survival of patients with cervical cancer $(\mathrm{P}>0.05)$ (Table 2).

The adjusted effect of each significant risk factor derived from univariable analysis were evaluated using the Cox proportional hazards regression model by forward stepwise of likelihood ratio (LR) with $\mathrm{PE}=0.1$ and $\mathrm{PR}$ $=0.15$. The results of the COX model showed that the adjusted relative risk of patients with a diagnostic age of 50 years or more compared to those aged less than 50 years was 1.978 .

The risk for married women aged less than 20 was 3.189 compared to aged 20 years or more. The adjusted relative risk of dead patients with grade II was approximately equal to grade I (1.004) and this risk was higher in patients with grade III and more compared to grade I (2.814). The adjusted relative risk of death in patients with recurrence was 6.186 times higher than those without recurrence (Table 3) (Figures 2-5).

\section{Discussion}

Cervical cancer is the fourth most common cancer among women in the world (World Health Organization, 2005). Some factors have been reported as important factors associated with survival in cervical cancer such as the high age of diagnosis, repeated infections with the human papillomavirus virus and histology of tumor (Federico et al., 2010; Hosseini et al., 2012; Roshany et al., 2012; Serur et al., 1995). In the present study, of the 109 patients, the mean (SD) time of diagnosis was obtained $50.1 \%$ (11.7) years. The mean (SD) and median of follow-up time was 38.23 (32.50) and 27.1 months (Mim:2 months and Max:132 months). The mean score of survival in patients with recurrence of 38.8 months was significantly lower than those without recurrence (102.5) $(\mathrm{P}<0.001)$. The adjusted relative risk of mortality with a diagnostic age of 50 years or more compared to those under 50 years of age was 1.978 . The risk for married women under age 20 was 3.189 compared to the marriage age of 20 years or more. 
In the present study with diagnosis age less than 50 years with mean survival time of 102.4 months was significantly different with 50 years and over (66.7 months), Serur et al., (1995) had a study on age, substance abuse, and survival of patients with cervical cancer performed on 1,173 patient records that had been recorded during 1967-1988. The results of this study showed that women over 70 years had a lower chance of survival compared to younger women at stage III. Drug abuse was common among younger patients, and patients who had smoking, substance abuse and alcohol, had lower survival rates, which was consistent with the present study.

In a study conducted by Christopher Federico et al., (2010) in Netherlands about the relationship among age, race, medical budget and cancer of the uterus in 2010 on 125 patients with cervical cancer, concluded that there was no relationship between race, medical education and the pattern of cancer screening and survival rate, but the age of diagnosis 65 or over that had a low chance of survival, so that the age factor was significantly associated with a reduction in survival probability, and were consistent with the present study. It should be noted that education is effective for promotion of cancer prevention behavior (Zareipour et al., 2018).

In the present study in terms of the type of cancer, 99 patients $(90.8 \%)$ were in the SCC group and 10 patients $(9.2 \%)$ were in the adenocarcinoma group. In the study of S Bulk et al., (2003) the survival rate of women with cervical cancer were evaluated, the results showed that SCC incidence have decreased while the incidence of adenocarcinoma was unchangeable. Indeed, patients with adenocarcinoma had lower survival rate compared to SCC that was related to histology of tumor, these results were inconsistent with the present study because there was no relationship between the type of cancer and survival rate in the present study.

In the study conducted by Razak et al., (2013) five-year survival among patients with cervical cancer treated in Malaysia were evaluated on 120 cervical cancer patients diagnosed during 1995-2007. The survival probability was determined using the Kaplan-Meier method and the log-rank test was applied to compare the survival distribution between groups. The overall five-year survival was $39.7 \%$ with a median survival time of 40.8 months. The log-rank test showed that there were survival differences between the groups for the: stage at diagnosis, which these results were consistent with the present study, so that the risk for married women aged less than 20 was 3.189 compared to aged 20 years or more. The adjusted relative risk of dead patients with grade II was approximately equal to grade I (1.004) and this risk was higher in patients with grade III and more compared to grade I (2.814).

As well as, in the study of Muhamad et al., (2015) they reported that data were collected during 2000-2005 and Kaplan Meier analyses were conducted to identify the overall survival rates and median survival time of patients with cervical cancer. A total of 5,859 patients were included. The median survival time for cervical cancer in this study was 65.8 months and the 5-year survival rate was $71.1 \%$. The overall observed survival rates at 1,3 and 5 years were $94.1 \%, 79.3 \%$ and $71.1 \%$ respectively. These results were consistent with the results of the present study so that estimates of survival rate of six months and one, three, five and seven years were 99\%, 96.9\%, 85.2\%, $73.1 \%$ and $57.7 \%$, respectively.

The present study was done on stage I, II and III and the adjusted relative risk of dead patients with grade II was approximately equal to grade I (1.004) and this risk was higher in patients with grade III and more compared to grade I (2.814). Lai et al., (2013) performed a study to assess the benefits of the 2009 International Federation of Gynecology and Obstetrics (FIGO) staging system for survival of patients with Stage IIA1 and IIA2 cervical cancer. A study cohort of 51 patients with Stage IIA 1 and 2 was retrospectively collected from the 2004-2009 hospital-based. The survivorship and overall survival were compared between these two groups (Stages IIA1 and IIA2) using log-rank test. Thirty-six and 15 patients were classified into Stages IIA1 and IIA2, respectively. Stage IIA2 patients were younger than those with Stage IIA1 disease but no significant difference was observed in confirmed pelvic lymph node status. Although the 2-year and 5-year overall survival was better among Stage IIA1 patients, there was no significant difference in survival between Stage IIA1 and IIA2 groups (2-year, 90.6\% vs. $77.8 \%$; 5-year, $86.3 \%$ vs. $51.9 \%, \mathrm{p}=0.218$ ).

These results in terms of survival rate between stages were approximately close to the results of the present study because there was no significantly difference between stage I and II.

Those who died before the end of the study or did not recover and excluded in any way during study raised the censored patients of the study, which was a kind of selection bias and can affect the validity of survival time among patients.

According to results, the mean survival time of patients with age more than 20 years was approximately 2 times (117.3 months) higher than patients with age less than 20 years (73.8 months). The low age of marriage increases the occurrence of advanced stages of cancer in older ages and as a social and cultural factor has a significant effect on the survival of patients with cervical cancer. Therefore, it should be considered important to improve the general awareness of people and coverage of health services in prevention and survival of patients with cervical cancer.

\section{Conflict of Interest}

The authors declared that there is no conflict of interest in this study.

\section{Acknowledgements}

This study was extracted from approved project with code 1394-0--63-1807 and the authors would like to thank the deputy of University research of Urmia for financial support, cooperation of health providers who participated in the completion of the sampling stages.

\section{References}

Bayrami R, Taghipour A, Ebrahimipour H (2015). Personal and 
socio-cultural barriers to cervical cancer screening in Iran, patient and provider perceptions: a qualitative study. Asian Pac J Cancer Prev, 16, 3729-34.

Bazaz M, Shahry P, Latifi SM, et al (2019). Cervical cancer literacy in women of reproductive age and its related factors. $J$ Cancer Educ, 34, 82-9.

Bulk S, Visser O, Rozendaal L, et al (2003). Incidence and survival rate of women with cervical cancer in the Greater Amsterdam area. Br J Cancer, 89, 834-9.

De Martel C, Ferlay J, Franceschi S, et al (2012). Global burden of cancers attributable to infections in 2008: a review and synthetic analysis. Lancet Oncol, 13, 607-15.

Federico C, Alleyn J, Dola C, et al (2010). Relationship among age, race, medical funding, and cervical cancer survival. J Natl Med Assoc, 102, 199-205.

Ferlay J (2004). (GLOBOCAN 2002) Cancer incidence, mortality and prevalence worldwide. IARC Cancer Base No. 5, version 2.0. http://www-depdb. iarc. fr/globocan/ GLOBOframe. htm.

Hosseini Teshnizi S, Zare SH, Tazhibi M (2012). The evaluation of Cox and Weibull proportional hazards models and their applications to identify factors influencing survival time in acute leukem. J Hormozgan Univ Med Sci, 15, 269-78.

Lai JY, Chou YJ, Huang N, et al (2013). Survival analysis of Stage IIA1 and IIA2 cervical cancer patients. Taiwanese $J$ Obstet Gynecol, 52, 33-8.

Muhamad NA, Kamaluddin MA, Adon MY, et al (2015). Survival rates of cervical cancer patients in Malaysia. Asian Pac J Cancer Prev, 16, 3067-72.

Razak NA, Khattak MN, Zubairi YZ, et al (2013). Estimating the five-year survival of cervical cancer patients treated in Hospital Universiti Sains Malaysia. Asian Pac J Cancer Prev, 14, 825-8.

Roshany D, Azadi NA, Esmailnasab N, et al (2011). Application of parametric, semiparametric and nonparametric approaches in survival analysis of patients with acute myocardial. $J N$ Khorasan Univ Med Sci, 3, 45-51.

Schmoll HJ, Van’t Meer L, Vermorken J, et al (2009). ESMO Handbook of cancer diagnosis and treatment evaluation. CRC Press.

Semnani S, Besharat S, Keshtkar AA, et al (2008). Short-term survival rates of upper gastrointestinal cancers in rural areas of golestan province, Iran. J Gorgan Univ Med Sci, 10, 51-7.

Serur E, Fruchter RG, Maiman M, et al (1995). Age, substance abuse, and survival of patients with cervical carcinoma. Cancer, 75, 2530-8.

Siegel RL, Miller KD, Jemal A (2015). Cancer statistics, 2015. CA Cancer J Clin, 65, 5-29.

Vaisy A, Lotfinejad S, Zhian F (2012). Relationship between utrine cervical carcinoma and oral contraceptives. J Gorgan Univ Med Sci, 14, 1-6.

World Health Organization (2002). Cervical cancer screening in developing countries: report of a WHO consultation. World Health Organization.

World Health Organization (2013). International Agency for Re-search on Cancer. IARC handbooks of cancer prevention Cervix Cancer Screening, 10.

Yazdanbod E, Samadi F, Malekzade R, et al (2005). Four-year survival rate of patients with upper GI cancer in ardabil. J Ardabil Univ Med Sci, 5, 180-4.

Zareipour MA, Mahmoodi H, Valizadeh R, et al (2018). impact of an educational intervention based on the BASNEF model on skin cancer preventive behavior of college students. Asian Pac J Cancer Prev, 16, 2717-22.

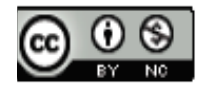

This work is licensed under a Creative Commons AttributionNon Commercial 4.0 International License. 